period many attempts, mostly abortive, have been made to deal with it. From 1934 research work with chemical methods has been carried out. These investigations were at first published in the Indian Forester but arenow reproduced with the figures in "The Control of Lantana by a Sodium Chlorate Spray", by A. L. Griffith, sylviculturist, Forest Research Institute, Dehra Dun (For. Bull., No. 106, Sylvicultural Series, Govt. of India Press, Calcutta, 1941). The importance of the investigations will be evident by the rapidity of the spread of the pest. In north Salem, where the work has been undertaken, it is recorded that in the north Salem forest division in 1917 there were 4,800 acres of Lantana. In 1935 the area had increased to 56,000 acres, while in 1941 it is stated that a very recent estimate put the area at just over 100,000 acres. This was the area in Government Reserved Forest only in the district. The density of the Lantana is often as much as 5,000 to 10,000 stems per acre. The experience gained so far shows that successful control by spraying with sodium chlorate is possible at a cost roughly of $£ 10$ per acre and that such a spray has no toxic effect on subsequent rejuvenation on the area. The results of this piece of research appear to be important. The Bulletin gives full details of the methods of applying the spray.

\section{Psychiatry and Alcoholism}

IN a recent paper (Virginia Med. Monthly, 69, 417 ; 1942), Dr. Merrill Moore, associate in psychiatry at the Harvard Medical School and director of research at the Washington Hospital for the Treatment and Prevention of Alcoholism, declares that the three greatest public health problems in the United States to-day are tuberculosis, syphilis and alcoholism. Although the first two have received continuous attention from the medical profession during the last twenty years, alcoholism has not yet been completely accepted as a medical problem. According to Dr. Moore, acute or chronic alcoholism seems to result partly from the psychological inadequacy of the individual, and is therefore most likely to be found in persons who in childhood have been over-protected, too strongly dominated or pampered by oversolicitous parents. Prolonged drinking usually results in some form of neuropsychiatric disorder, which may be an avitaminosis, such as alcoholic neuritis or pellagra caused by faulty diet, or there may be a frank alcoholic psychosis such as Korsakow's syndrome.

The frequent association of mental disease with alcoholism is shown by the fact that 60 per cent of all patients admitted to Massachusetts mental hospitals in the last twenty-five years gave a history of alcoholism. The high cost of alcoholism to the taxpayer is due to the fact that most alcoholic men and women are cared for in State or municipal hospitals to which they make no payment, and relief is re quired for the families and dependants of alcoholics whose income and earning power have been lost. Moreover, the cost is increased by the maintenance of courts for the distribution of drunkards in penal institutions. Dr. Moore maintains that there is a great need for the establishment of special clinics and hospital facilities for the treatment of alcoholics. At present they are not welcomed for treatment unless they can afford the necessarily high cost of private institutions, nor are they accepted at most State institutions unless they are suffering from alcoholic psychoses or equally severe mental illnesses in which alcoholism plays a secondary part.

\section{The Journey of the Sun}

RALF E. Witson discusses the motion of the sun in the Galaxy in Leaflet No. 161 (July, 1942) issued by the Astronomical Society of the Pacific. Before 1900, all determinations of the apex of the sun's motion were based upon proper motions, that is, motions at right angles to the line joining the star to the earth; and many of these were poorly deter. mined and affected by appreciable systematic errors. About 1910, previous determinations were superseded by the results of two major investigations. The proper motions of about 6,000 stars had been determined on a standard system, and the observations started early in the century had produced 1,200 radial velocities. The latter gave an apex about $9^{\circ}$ south of that derived from proper motions, and part of the discrepancy was attributed to the fact that radial velocities referred almost entirely to stars brighter than magnitude $5 \cdot 5$, the proper motions including essentially all stars brighter than magnitude 6 and many fainter ones. As radial velocities became available for the fainter stars the discordance increased, and in 1925 was largely removed by the discovery of systematic errors in the proper motions. The most recent investigations show that the motion of the sun, with respect to the naked-eye stars, is towards a point at right ascension $18 \mathrm{~h}$. and declination $+30^{\circ}$, near o Herculis, with a speed of 12 miles a second. The actual direction in which the sun is travelling in its galactic orbit lies about $40^{\circ}$ north and east of the position just given, and the speed is about 180 miles a second. The low-velocity stars, with reference to which the sun's speed is 12 miles a second, are members of the same galactic subsystem as the sun, and are also subject to the same amount of rotation. The rotation of the sun around the centre of the galaxy is completed in about 200 million years.

\section{New Companion to $\mu$ Draconis}

Dr, K. Aa. Strand, of Sproul Observatory, has reported the existence of a third companion in the double-star system of $\mu$ Draconis (Sky and Telescope, August). It is known only by the regular fluctuations from the expected motion of the two visible components, observations having extended over three years. The brighter components have a period of revolution of about 1,500 years and the new companion revolves around one of them at a distance of about three astronomical units. It is a dwarf star with a mass half that of the sun.

\section{Electricity in the Annealing of Glass}

A PAPER by E. S. Morris on the application of electricity to the annealing of glass bottles and containers (J. Inst. Elec. Eng., 89, Pt. 2, No. 10 ; Aug., 1942) first explains the nature and primary importance of annealing processes as applied to commercial glassware, with particular reference to bottles and containers. Development stages, from heated brick arches to portable and continuous lehrs, are then described in the section on mechanical improvements. The application of controlled heat to the fused and moulded product is shown through the transition stages of coal, producer-gas and oil firing, to complete electrification of annealing lehrs. Insulation factors and methods; heat conservation and re-application; cooling graduations; economic and product quality considerations in favouring the application of electricity as a power and heating element, are all made 